\title{
FINITE GROUPS WHOSE IRREDUCIBLE BRAUER CHARACTERS HAVE PRIME POWER DEGREES
}

BY

\author{
Hung P. TONG-VIET \\ School of Mathematics, Statistics and Computer Science \\ University of KwaZulu-Natal \\ Pietermaritzburg 3209, South Africa \\ e-mail: Tongviet@ukzn.ac.za
}

\begin{abstract}
Let $G$ be a finite group and let $p$ be a prime. In this paper, we classify all finite quasisimple groups in which the degrees of all irreducible $p$-Brauer characters are prime powers. As an application, for a fixed odd prime $p$, we classify all finite nonsolvable groups with the above mentioned property and having no nontrivial normal $p$-subgroups. Furthermore, for an arbitrary prime $p$, a complete classification of finite groups in which the degrees of all nonlinear irreducible $p$-Brauer characters are primes is also obtained.
\end{abstract}

\section{Introduction}

Throughout this paper, $G$ is a finite group and $p$ is a fixed prime. The study of finite groups all of whose complex irreducible characters have prime power degrees is a subject of interest for quite some time dating back to papers of Isaacs and Passman first published in 1965. In [12, 13], among other things, the authors investigated in detail the structure of finite groups whose all nonlinear complex irreducible characters have prime degrees. In particular, such finite groups $G$ are solvable and the set of primes which divide some character degree of $G$ has cardinality at most two. These results have been improved by $\mathrm{O}$. Manz in $[20,21]$, where he showed that if the degrees of all complex irreducible characters of a finite group $G$ are prime powers, then either $G$ is solvable and there are at most two primes which can divide some character degree of $G$ or 
$G$ is nonsolvable and $G=S \times A$, where $S \cong \operatorname{PSL}_{2}(q)$ with $q \in\{5,8\}$ and $A$ is an abelian group (see also [22, Corollary]). These results have led to the following famous conjecture known as Huppert's $\rho-\sigma$ conjecture which says that $|\rho(G)| \leq 2 \sigma(G)$ if $G$ is solvable; and $|\rho(G)| \leq 3 \sigma(G)$ in general, where $\rho(G)$ is the set of all primes which divide some complex character degree of $G$ and $\sigma(G)$ is the largest number of distinct primes that divide any given complex character degree of $G$. (See [10]). Recent results concerning this conjecture can be found in [2].

We now turn our attention to $p$-Brauer characters. Let $\operatorname{IBr}_{p}(G)$ denote the set of all irreducible $p$-Brauer characters of $G$ and let $\operatorname{cd}_{p}(G)$ be the set of all degrees of irreducible $p$-Brauer characters of $G$. We write $\rho_{p}(G)$ for the set of all primes which divide the degree of some irreducible $p$-Brauer character of $G$ and $\sigma_{p}(G)$ for the maximal number of distinct primes which can divide any given irreducible $p$-Brauer character degree of $G$. We want to study a similar problem for $p$-Brauer characters of finite groups, that is, when all irreducible $p$-Brauer characters of a finite group $G$ have prime power degrees, in other words, when $\sigma_{p}(G)=1$. In this situation, the problem is much more difficult as the degree of an irreducible $p$-Brauer character of a group $G$ may not divide the order of $G$. Furthermore, several important results in characteristic 0 do not hold in characteristic $p$. For example, Thompson's theorem [11, Corollary 12.2] which states that if a prime $r$ divides the degrees of all nonlinear complex irreducible characters of a finite group $G$, then $G$ has a normal $r$-complement does not hold for Brauer characters. In fact, there are nonabelian simple groups whose all irreducible 2-Brauer characters have 2-power degrees. Also, the celebrated Ito-Michler's theorem [23, Theorem 5.5] only holds for the prime $p$, i.e., the finite group $G$ has a normal Sylow $p$-Subgroup if and only if the degrees of all irreducible $p$-Brauer characters of $G$ are coprime to $p$. Another difficulty is that the degrees of irreducible $p$-Brauer characters of nonabelian simple groups are still unknown. Recall that a finite group $G$ is a quasisimple group if $G$ is perfect and $G / \mathrm{Z}(G)$ is a nonabelian simple group. There has been some interest in classifying quasisimple groups $G$ with $\sigma_{p}(G)=1$. For instance, Tiep and Willems [26] classified all quasisimple groups $G$ in which the degrees of all irreducible $p$-Brauer characters are powers of a fixed prime. Bessenrodt and Weber [1] obtained a classification of all alternating groups $\mathrm{A}_{n}$ of degree $n \geq 1$ with $\sigma_{p}\left(\mathrm{~A}_{n}\right)=1$. In our first result, we complete this classification by proving the following result. 
Theorem A: Let $G$ be a finite quasisimple group and $p$ be a prime. If all $p$ Brauer irreducible characters of $G$ have prime power degrees, then the following hold:

(1) $G / \mathrm{Z}(G) \cong \mathrm{PSL}_{2}\left(2^{f}\right)$ with $f \geq 2$, $\mathrm{PSp}_{4}\left(2^{f}\right)$ with $f \geq 2,{ }^{2} \mathrm{~B}_{2}\left(2^{2 f+1}\right)$ with $f \geq 1, \mathrm{PSL}_{3}\left(2^{f}\right)$ with $1 \leq f \leq 2$, or $\mathrm{PSL}_{2}(q)$ with $q \geq 17$ a Fermat prime; $p=2$ and $\mathrm{Z}(G)$ is a $p$-group,

(2) $G / \mathrm{Z}(G) \cong \mathrm{A}_{6}$ and $p=2$,

(3) $G \cong \operatorname{PSL}_{2}\left(2^{f}\right)$ where $f \geq 5$ is prime and $p=2^{f}-1$ is a Mersenne prime,

(4) $G \cong \mathrm{A}_{5}$ or $\mathrm{PSL}_{2}(8)$ and $p$ is an odd prime,

(5) $G \cong \mathrm{PSL}_{2}\left(p^{f}\right)$ with $p$ odd and $7 \leq p^{f} \leq 13$,

(6) $G \cong \mathrm{SL}_{2}(5) \cong 2 \cdot \mathrm{A}_{5}$ and $p=5$,

(7) $G \cong 3 \cdot \mathrm{A}_{6}$ and $p=3$.

The proof of this theorem will be carried out in Section 3. Most part of that section is devoted to classifying finite simple groups $G$ with $\sigma_{p}(G)=1$. The corresponding results for quasisimple groups follow immediately. In view of [1], we will focus only on finite simple groups of Lie type and sporadic simple groups. This is achieved in Theorem 3.4. The classification for sporadic simple groups is done by using GAP [5] and results of Jansen [14]. For finite simple groups of Lie type in characteristic $r$, we consider the cases when $r=p$ and $r \neq p$ separately. For the former case, we use some results on the representation theory of finite groups of Lie type in defining characteristic given in Lübeck [17]. For the latter case, we apply results of Malle [18] on the defect zero graphs of finite simple groups.

Extending results of Isaacs and Passman in $[12,13]$ to positive characteristic, B. Huppert [9] proved that if $G$ is a finite $p$-solvable group with $\mathbf{O}_{p}(G)=1$ and that the degrees of all nonlinear irreducible $p$-Brauer characters of $G$ are primes and assume further that $G$ is not one of the groups in [12, Theorem C 4.8] nor [13, Theorem 6.1] then $p=3, G^{\prime} \cong \mathrm{SL}_{2}(3)$ and $\left|G: G^{\prime} \mathrm{Z}(G)\right|=2$. In our next theorem, we generalize this result to finite nonsolvable groups when $p$ is an odd prime.

Theorem B: Let $G$ be a finite nonsolvable group and let $p$ be an odd prime. Suppose that $\mathbf{O}_{p}(G)=1$ and that all irreducible $p$-Brauer characters of $G$ have 
prime power degrees. Then $G^{\prime}$ is a quasisimple group and one of the following holds:

(a) $G^{\prime}$ is a simple group, $\mathrm{C}_{G}\left(G^{\prime}\right)=\mathrm{Z}(G)$ is an abelian $p^{\prime}$-group and one of the following cases holds:

(i) $G \cong G^{\prime} \times \mathrm{Z}(G)$, where $G^{\prime}$ and $p$ are given in Cases (3) - (5) of Theorem $A$,

(ii) $G / \mathrm{Z}(G) \cong \mathrm{PSL}_{2}(8) \cdot 3, G^{\prime} \cong \mathrm{PSL}_{2}(8)$ and $p=3$,

(iii) $G / \mathrm{Z}(G) \cong \mathrm{PGL}_{2}\left(p^{f}\right), G^{\prime} \cong \mathrm{PSL}_{2}\left(p^{f}\right)$, where $p^{f}$ is odd and $5 \leq$ $p^{f} \leq 13$,

(b) $G^{\prime} \cong \mathrm{SL}_{2}(5), p=5, \mathrm{C}_{G}\left(G^{\prime}\right) / \mathrm{Z}\left(G^{\prime}\right)$ is an abelian $p^{\prime}$-group, the degree of every irreducible $p$-Brauer character of $\mathrm{C}_{G}\left(G^{\prime}\right)$ is a power of 2 , and either $G=\mathrm{C}_{G}\left(G^{\prime}\right) G^{\prime}$ is a central product or $G / \mathrm{C}_{G}\left(G^{\prime}\right) \cong \mathrm{PGL}_{2}(5)$.

Conversely, if a group $G$ and a prime $p$ satisfy one of the conditions above, then all irreducible $p$-Brauer characters of $G$ have prime power degrees.

Since $\mathbf{O}_{p}(G)$, the largest normal $p$-subgroup of $G$, is contained in the kernel of every irreducible $p$-Brauer character of $G$, it is reasonable to assume that $\mathbf{O}_{p}(G)=1$ in the statement of Theorem B. This theorem will be proved in Section 4. Notice that if $G$ is a finite group, $p$ is a prime and $N \unlhd G$ such that $\sigma_{p}(G)=1$, then both $\sigma_{p}(N)$ and $\sigma_{p}(G / N)$ are trivial. The most crucial step in the proof of Theorem B is the classification of finite perfect groups $G$ with $\sigma_{p}(G)=1$. In fact, it is proved in Theorem 4.2 that if $G$ is a finite perfect group with $\sigma_{p}(G)=1$ and $\mathbf{O}_{p}(G)=1$, then $G$ is a quasisimple group and the possibilities for $G$ and $p$ are given in Cases $(3)-(6)$ of Theorem A. We are unable to obtain a similar result in the case $p=2$ since $\rho_{2}(G)=\{2\}$ for almost all quasisimple groups $G$ with $\sigma_{2}(G)=1$. This is exactly where our argument fails (see the proof of (1) of Theorem 4.2). Notice that if $G$ is quasisimple and $\sigma_{p}(G)=1$ with $p$ an odd prime, then $\left|\rho_{p}(G)\right| \geq 2$.

In the last result, we complete the classification of finite groups whose all nonlinear irreducible $p$-Brauer characters have prime degrees.

Theorem C: Let $G$ be a finite group and let $p$ be a prime. Suppose that $\mathbf{O}_{p}(G)=1$ and that the degrees of all nonlinear irreducible $p$-Brauer characters of $G$ are primes. Then the following hold:

(1) $G$ is solvable and the degrees of all complex irreducible characters of $G$ are 1 or prime; 
(2) $G$ is solvable, $G^{\prime} \cong \mathrm{SL}_{2}(3)$ and $\left|G: \mathrm{Z}(G) G^{\prime}\right|=2$ with $p=3$;

(3) $G$ is nonsolvable, $G^{\prime} \cong \mathrm{PSL}_{2}(p)$ with $p \in\{5,7\}$ and $\left|G: G^{\prime} \mathrm{Z}(G)\right| \leq 2$.

Finally, we mention that it is proved in [15] that if $G$ is a solvable group with $\mathbf{O}_{p}(G)=1$ and $\sigma_{p}(G)=1$, then $\left|\rho_{p}(G)\right| \leq 2$. For nonsolvable groups $G$ with $\mathbf{O}_{p}(G)=1$ and $\sigma_{p}(G)=1$, where $p$ is an odd prime, Theorem B implies that $\left|\rho_{p}(G)\right| \leq 5$ and this bound is best possible as $\left|\rho_{p}(H)\right|=5$ and both $\sigma_{p}(H)$ and $\mathbf{O}_{p}(H)$ are trivial, where $H \cong \mathrm{PSL}_{2}(13)$ and $p=13$.

Notation: All groups in this paper are finite. We follow the notation in [11] for complex characters and in [24] for $p$-Brauer characters. Let $G$ be a finite group and let $p$ be a prime. We write $\operatorname{Irr}(G)$ for the set of all complex irreducible characters of $G$ and let $\operatorname{cd}(G)=\{\chi(1) \mid \chi \in \operatorname{Irr}(G)\}$. Similarly, $\operatorname{IBr}_{p}(G)$ is the set of all irreducible $p$-Brauer characters of $G$ and $\operatorname{cd}_{p}(G)=\left\{\varphi(1) \mid \varphi \in \operatorname{IBr}_{p}(G)\right\}$. If $N \unlhd G$ and $\lambda \in \operatorname{IBr}_{p}(N)$, then $\operatorname{IBr}_{p}(G \mid \lambda)$ is the set of all constituents of $\lambda^{G}$ in $\operatorname{IBr}_{p}(G)$. Furthermore, $\operatorname{IBr}_{p}(G \mid N)=\left\{\varphi \in \operatorname{IBr}_{p}(G) \mid N \nsubseteq \operatorname{ker}(\varphi)\right\}$. If $\chi \in \operatorname{Irr}(G)$, then $\chi^{\circ}$ is the restriction of $\chi$ to $G^{\circ}$, the set of all $p$-regular elements of $G$. The trivial $p$-Brauer character of $G$ is denoted by $1_{G^{\circ}}$. The set of all prime divisors of the order of $G$ is denoted by $\pi(G)$. A group $G$ is said to be a central product of two normal subgroups $H$ and $K$ if $G / Z=H / Z \times K / Z$ and $[H, K]=1$, where $Z=H \cap K$. Other notation is standard.

\section{Preliminaries}

Recall that a complex irreducible character $\chi \in \operatorname{Irr}(G)$ is said to have $p$-defect zero for some prime $p$ if $\chi(1)_{p}=|G|_{p}$. It is well known that if $\chi \in \operatorname{Irr}(G)$ is of $p$-defect zero, then $\chi^{\circ} \in \operatorname{IBr}_{p}(G)$ (see [24, Theorem 3.18]). Following Malle [18], the defect zero graph $\Delta^{\circ}(G)$ of a group $G$ is a graph whose vertex set is the set of all primes dividing $|G|$ and two distinct vertices $u, v$ are connected in $\Delta^{\circ}(G)$ if there exists a complex irreducible character $\chi \in \operatorname{Irr}(G)$ which is of defect zero for both $u$ and $v$. (We reserve the notation $\Delta(G)$ for the prime graph of a group $G$ which will be defined in Section 4.) Now let $S$ be a simple group of Lie type in characteristic $r$ with $S \neq{ }^{2} \mathrm{~F}_{4}(2)^{\prime}$. It is well known that the Steinberg character of $S$, denoted by $\mathrm{St}_{S}$, of degree $|S|_{r}$ is the only complex irreducible character of $S$ of $r$-defect zero. Hence, the defining prime $r$ is an isolated vertex in the defect zero graph $\Delta^{\circ}(S)$. 
Lemma 2.1: Let $S$ be a nonabelian simple group of Lie type defined over a finite field of size $q$ in characteristic $r$ with $S \neq{ }^{2} \mathrm{~F}_{4}(2)^{\prime}$. Then the defect zero graph $\Delta^{\circ}(S)$ of $S$ has two connected components, one of which consists only the defining prime $r$, or $S$ is one of the following groups:

(1) $\operatorname{PSL}_{2}(q)$ with $q \geq 4$,

(2) $\operatorname{PSL}_{3}(q), q=2^{6 m+1}-1$ with $6 m+1$ prime or $q=3$,

(3) $\operatorname{PSU}_{3}(q), q=2^{2^{2 m}}+1$ with $m \geq 0$ or $q=9$,

(4) $\operatorname{PSp}_{4}(q)$ with $q \in\{3,5\}$.

Proof. This follows from Propositions 2.4 and 2.5 in [18].

The following result due to Zsigmondy will be very useful.

Lemma 2.2: (Zsigmondy's theorem [27]). Let $p$ be a prime and let $n \geq 2$ be an integer. Then there exists a prime $\ell$ such that $\ell \mid p^{n}-1$ and $\ell$ does not divide $p^{m}-1$ for $1 \leq m<n$ unless

(1) $p=2$ and $n=6$ or

(2) $p=2^{r}-1$ is a Mersenne prime and $n=2$, with $r$ being a prime.

As an easy application of Zsigmondy's theorem, we obtain the following result.

Lemma 2.3: Let $p$ and $r$ be primes and $a, f \geq 1$ be integers such that $p^{f}=$ $1+r^{a}$. Then one of the following cases holds:

(1) $r=2, p=3, f=2$ and $a=3$,

(2) $r=2, f=1, a$ is a 2-power and $p=2^{a}+1$ is a Fermat prime,

(3) $p=2, a=1$ and $r=2^{f}-1$ is a Mersenne prime, with $f$ being a prime.

The following result is well known, see for example [1, Proposition 6.1]

Lemma 2.4: Let $n \geq 1$ be an integer. Then a binomial $\left(\begin{array}{l}n \\ k\end{array}\right)$ with $0 \leq k \leq n$ is a nontrivial prime power only in the cases when $k=1$ or $n-1$ and $n$ is a prime power.

\section{Simple groups with all irreducible $p$-Brauer characters having prime power degrees}

In this section, we classify all nonabelian simple groups $S$ whose all irreducible $p$ Brauer characters have prime power degrees. The case when $S$ is an alternating group has been handled by Bessenrodt and Weber [1]. Therefore, we only need 
to consider the sporadic simple groups and the simple groups of Lie type. Notice that we consider the Tits group as a sporadic simple group rather than a simple group of Lie type. We begin with the following observation.

LEMma 3.1: Let $S$ be a nonabelian simple group and let $p$ be a prime. Suppose that $\sigma_{p}(S)=1$. Then the following hold:

(1) If $p \notin \pi(S)$, then $S \cong \mathrm{PSL}_{2}(q)$ with $q \in\{4,5,8\}$.

(2) If $p \in \pi(S)$ and $S$ has an irreducible character $\chi \in \operatorname{Irr}(S)$ of $p$-defect zero, then $\chi^{\circ} \in \operatorname{IBr}_{p}(S), \chi^{\circ}(1)=\chi(1)=|S|_{p}$ and $p$ is an isolated vertex of the defect zero graph $\Delta^{\circ}(S)$.

Proof. By [24, Theorem 2.12], we have that $\operatorname{Irr}(S)=\operatorname{IBr}_{p}(S)$ whenever $p \nmid|S|$. Thus, if $p \notin \pi(S)$, then all complex irreducible characters of $S$ have prime power degrees. By [22, Corollary] we obtain that $S \cong \mathrm{PSL}_{2}(4) \cong \mathrm{PSL}_{2}(5)$ or $\mathrm{PSL}_{2}(8)$. This proves (1). Assume that $p \in \pi(S)$. By [24, Theorem 3.18], if $\chi \in \operatorname{Irr}(S)$ has $p$-defect zero, then $\chi^{\circ} \in \operatorname{IBr}_{p}(S)$ and so $\chi^{\circ}(1)=\chi(1)$ is a prime power. Since $\chi(1)_{p}=|S|_{p}>1$, we must have that $\chi(1)=|S|_{p}$. In particular, all irreducible complex characters of $S$ of $p$-defect zero must have the same degree $|S|_{p}$. Hence, $p$ must be an isolated vertex in the defect zero graph $\Delta^{\circ}(S)$. This completes the proof.

For the next result, we consider the following set up. Let $\mathscr{G}$ be a simply connected simple algebraic group and let $F: \mathscr{G} \longrightarrow \mathscr{G}$ be a suitable Frobenius map such that $S \cong \mathscr{G}^{F} / \mathrm{Z}\left(\mathscr{G}^{F}\right)$ is a finite nonabelian simple group of Lie type defined over a finite field of size $q=p^{f}$ in characteristic $p$, and that $S \neq{ }^{2} \mathrm{~F}_{4}(2)^{\prime}$. We refer to [17] for some terminology and notation on the representation theory of finite groups of Lie type in defining characteristic. Let $\ell$ be the Lie rank of $\mathscr{G}$ and fix a maximal torus $T$ of $\mathscr{G}$. Let $X$ be the character group of $T$ and let $\left\{\alpha_{1}, \alpha_{2}, \cdots, \alpha_{\ell}\right\} \subseteq X$ be a set of simple roots for $\mathscr{G}$ with respect to $T$. Let $\left\{\omega_{1}, \omega_{2}, \cdots, \omega_{\ell}\right\}$ be the fundamental weights. A weight $\omega \in X$ is called dominant if it is a non-negative linear combination of fundamental weights. If $q$ is a power of $p$, then we put $X_{q}=\left\{\sum_{i=1}^{\ell} a_{i} \omega_{i} \mid a_{i} \in \mathbb{Z}, 0 \leq a_{i} \leq q-1\right\}$ if $\mathscr{G}^{F}$ is not a Suzuki or Ree group; for Suzuki and Ree groups, we define $X_{q}=\left\{\sum_{i=1}^{\ell} a_{i} \omega_{i} \mid 0 \leq a_{i} \leq q / \sqrt{p}-1\right.$ if $\alpha_{i}$ is a long root, $0 \leq a_{i} \leq q \sqrt{p}-$ 1 if $\alpha_{i}$ is a short root\}. A dominant weight $\lambda=a_{1} \omega_{1}+a_{2} \omega_{2}+\cdots+a_{\ell} \omega_{\ell} \in X$ is called $p$-restricted if $0 \leq a_{i} \leq p-1$. It is well known that for each dominant weight $\lambda \in X$, there is an irreducible $\mathscr{G}$-module $L(\lambda)$ with highest weight $\lambda$ 
(see [17, Theorem 2.1]). By Steinberg's tensor product theorem, all highest weight modules $L(\lambda)$ of $\mathscr{G}$ can be constructed from modules with $p$-restricted highest weights. Then all the representations of $\mathscr{G}^{F}$ is obtained by restricting $\mathscr{G}_{-}$ modules $L(\lambda)$ with $\lambda \in X_{q}$ to $\mathscr{G}^{F}$. (See [17, Theorem 2.2, 2.3]). If $n$ is an integer and $p$ is a prime, then we define $\epsilon_{p}(n)=1$ if $p \mid n$ and $\epsilon_{p}(n)=0$, otherwise. For $\epsilon= \pm$, we use the convention that $\operatorname{PSL}_{n}^{\epsilon}(q)$ is $\operatorname{PSL}_{n}(q)$ if $\epsilon=+$ and $\operatorname{PSU}_{n}(q)$ if $\epsilon=-;$ similar convention applies to $\mathrm{SL}_{n}^{\epsilon}(q)$. We also write $\mathrm{E}_{6}^{+}(q)$ for $\mathrm{E}_{6}(q)$ and $\mathrm{E}_{6}^{-}(q)$ for ${ }^{2} \mathrm{E}_{6}(q)$. Also, recall that $\mathrm{A}_{5} \cong \operatorname{PSL}_{2}(4) \cong \operatorname{PSL}_{2}(5)$, $\mathrm{PSL}_{2}(9) \cong \mathrm{PSp}_{4}(2)^{\prime} \cong \mathrm{A}_{6}$ and $\mathrm{PSL}_{4}(2) \cong \mathrm{A}_{8}$.

LEMMA 3.2: Let $S$ be a nonabelian simple group of Lie type defined over a field of size $q$ with $q=p^{f}$ and $S \neq{ }^{2} \mathrm{~F}_{4}(2)^{\prime}$. Suppose that $\sigma_{p}(S)=1$ and that $S$ is not isomorphic to an alternating group nor $\mathrm{PSL}_{2}(8)$. Then one of the following cases holds:

(1) $S \cong \mathrm{PSL}_{2}\left(2^{f}\right)$ with $f \geq 4, \mathrm{PSp}_{4}\left(2^{f}\right)$ with $f \geq 2,{ }^{2} \mathrm{~B}_{2}\left(2^{2 f+1}\right)$ with $f \geq 1$, $\mathrm{PSL}_{3}\left(2^{f}\right)$ with $1 \leq f \leq 2$, or

(2) $S \cong \mathrm{PSL}_{2}(p)$ with $p$ odd and $7 \leq p \leq 13$,

Proof. Let $Z$ be the center of $G(q):=\mathscr{G}^{F}$ and let $\ell$ be the Lie rank of $\mathscr{G}$. Then $S \cong G(q) / Z$. We consider each type of $\mathscr{G}$ separately.

(1) $\mathscr{G}$ is of type $\mathrm{A}_{\ell}, \ell \geq 1$. Then $S \cong \operatorname{PSL}_{\ell+1}^{\epsilon}(q)$ with $\epsilon= \pm$. By [18, Lemma 3.7], $S$ has irreducible $p$-Brauer characters of degree $\ell^{2}+2 \ell-\epsilon_{p}(\ell+1)$ and $\left(\begin{array}{c}\ell+1 \\ k\end{array}\right)$ if $k$ is a multiple of $d:=\operatorname{gcd}(\ell+1, q-\epsilon 1)$. These are the dimensions of irreducible representations of $G(q)$ with highest weights $\omega_{1}+\omega_{\ell}$ and $\omega_{k}$, respectively.

Case $\ell \geq 3$. Assume first that $p \nmid \ell+1$. Then $\ell^{2}+2 \ell \in \operatorname{cd}_{p}(S)$ and thus $\ell(\ell+2)=r^{a}$ for some prime $r$ and some integer $a \geq 1$. Since $\operatorname{gcd}(\ell+2, \ell)=$ $\operatorname{gcd}(2, \ell)$, it must be that $r=2$ and hence $\ell=2^{u}$ for some $u \geq 2$ since $\ell \geq 3$. It follows that $\ell+2=2^{u}+2=2^{a-u}$ and thus $2^{u-1}+1=2^{a-u-1}$. Since $u-1 \geq 1$, the previous equation is impossible. Assume now that $p \mid \ell+1$. Since $p \nmid q-\epsilon 1$, we deduce that $\ell+1 \nmid q-\epsilon 1$ and thus $d=\operatorname{gcd}(\ell+1, q-\epsilon 1)<\ell+1$. Let $k=\max \{2, d\}$. Since $\ell \geq 3$, we obtain that $d=\operatorname{gcd}(\ell+1, q-\epsilon 1) \neq \ell$ and so $2 \leq k<\ell$. Hence, $\left(\begin{array}{c}\ell+1 \\ k\end{array}\right)$ is the degree of an irreducible $p$-Brauer character of $S$ and thus it must be a nontrivial prime power, which is impossible by Lemma 2.4 .

Case $\ell=2$. Assume first that $3 \nmid q-\epsilon 1$. Then the center $Z$ of $G(q) \cong$ $\mathrm{SL}_{3}^{\epsilon}(q)$ is trivial. Now if $p \geq 3$, then $L\left(2 \omega_{2}\right)$, with $2 \omega_{2}$ being a $p$-restricted 
dominant weight, has dimension $\left(\begin{array}{c}2+2 \\ 2\end{array}\right)=6$, which is not a prime power (see [17, Appendix A.6]). Assume that $p=2$. Since $3 \nmid q-\epsilon 1$, we must have that $q=2^{2 m+1}$ if $\epsilon=+$, and $q=2^{2 m}$ if $\epsilon=-$. Using [5], we can check that $\mathrm{PSL}_{3}(2) \cong \mathrm{PSL}_{2}(7)$ satisfies the hypothesis of the lemma and since $\mathrm{PSU}_{3}(2)$ is not simple, we can assume that $q>2$ and hence $m \geq 1$. In this case, by [17, Appendix A.6] the adjoint module $L\left(\omega_{1}+\omega_{2}\right)$ has dimension 8 and the natural module $L\left(\omega_{2}\right)$ has dimension 3, and thus by Steinberg's tensor product theorem [17, Theorem 2.2], we have that $L\left(\left(\omega_{1}+\omega_{2}\right)+2 \omega_{2}\right)$ has dimension $8 \cdot 3=24$, which is not a prime power. Assume that $3 \mid q-\epsilon 1$. It follows that $p \neq 3$ and $|Z|=3$. Let $\xi \in \mathbb{F}_{p}^{*}$ be an element of order 3 and let $z$ be a generator of $Z$. Then $\left(\omega_{1}(z), \omega_{2}(z)\right)=\left(\xi, \xi^{2}\right)$ by [17, Appendix A.2]. If $p \geq 5$, then $L\left(3 \omega_{1}\right)$ has dimension $\left(\begin{array}{c}2+3 \\ 3\end{array}\right)=10$ and $Z$ acts trivially on this module. Hence, $L\left(3 \omega_{1}\right)$ is an irreducible $S$-module and so $10 \in \operatorname{cd}_{p}(S)$, which is a contradiction. Assume that $p=2$. Then we have that $q=2^{2 m}$ if $\epsilon=+$, and $q=2^{2 m+1}$ if $\epsilon=-$. Using [5], we can check that $\mathrm{PSL}_{3}(4)$ satisfies the hypothesis of the lemma while $\mathrm{PSU}_{3}(8)$ does not. Hence, we can assume that $m \geq 2$. We can see that $L\left(\left(\omega_{1}+\omega_{2}+2 \omega_{2}+4 \omega_{2}\right)\right)$ admits a trivial action of $Z$ and has dimension $8 \cdot 3^{2}=72$. Hence, $72 \in \operatorname{cd}_{p}(S)$, which is impossible.

Case $\ell=1$. Then $S \cong \mathrm{PSL}_{2}(q)$ with $q=p^{f}$. If $p=2$, then all the irreducible $p$-modular characters of $S$ have 2-power degrees by [26, Proposition 2.4]. Assume now that $p$ is odd. If $q=p \geq 7$, then $\operatorname{cd}_{p}\left(\operatorname{PSL}_{2}(q)\right)$ contains all the odd numbers from 1 to $p$ by [17, Remark 4.5]. By the hypothesis, we deduce that $q=p \in\{7,11,13\}$. Assume that $q=p^{f}$ with $p \geq 3$ and $f \geq 2$. Assume first that $p \geq 5$. Then the dimensions of the representations for the $p$-restricted weights $2 \omega_{1}$ and $4 \omega_{1}$ are 3 and 5 , respectively. By applying Steinberg's tensor product theorem [17, Theorem 2.2], we deduce that the dimension of the representation $L\left(2 \omega_{1}+3 \cdot\left(4 \omega_{1}\right)\right)$ is 15 , which is not a prime power. Furthermore, the center $Z$ of $\mathrm{SL}_{2}(q)$ acts trivially on this representation, so $15 \in \operatorname{cd}_{p}(S)$, which is a contradiction. Assume now that $p=3$. Then $f \geq 3$. By Steinberg's tensor product theorem [17, Theorem 2.2], the dimension of the representation $L(\lambda)$ with $\left.\lambda=\omega_{1}+3 \omega_{1}+3^{2}\left(2 \omega_{1}\right)\right)$ is $2 \cdot 2 \cdot 3=12$ and $Z$ acts trivially on $L(\lambda)$ by applying [17, Appendix A.2]. Hence, $12 \in \operatorname{cd}_{3}(S)$, a contradiction.

(2) $\mathscr{G}$ is of type $C_{\ell}, \ell \geq 2$. We have that $|Z|=\operatorname{gcd}(2, p-1)$. If $(\ell, p)=(2,2)$, then all irreducible 2-Brauer characters of $\operatorname{Sp}_{4}\left(2^{f}\right), f \geq 2$ or ${ }^{2} \mathrm{~B}_{2}\left(2^{2 f+1}\right), f \geq 1$, have 2-power degrees by [26, Proposition 2.4]. Hence, we can assume that $(\ell, p) \neq(2,2)$. Assume that $p \geq 3$. Then $2 \omega_{\ell}$ is $p$-restricted and $Z$ acts trivially 
on $L\left(2 \omega_{\ell}\right)$ by [17, Appendix A.2] and so by [17, Table 2], $2 \ell^{2}+\ell=\operatorname{dim}\left(L\left(2 \omega_{\ell}\right)\right)$ must be a prime power. However this is impossible since $\operatorname{gcd}(\ell, 2 \ell+1)=1$ and $\ell \geq 2$. Assume next that $p=2$ and $\ell \geq 3$. In this case, the center of $G$ is trivial and thus by applying [17, Table 2], $S$ has irreducible $p$-Brauer characters of degree $2 \ell$ and $2 \ell^{2}-\ell-1-\epsilon_{2}(\ell)$. In all cases, we see that these degrees are even and thus they must be 2-powers. It follows that $\ell=2^{k}$ for some $k \geq 2$ and $2 \ell^{2}-\ell-1-\epsilon_{2}(\ell)=2^{a}$ for some $a \geq 2$. We now have that $2^{2 k+1}-2^{k}-2=2^{a}$ and so $2^{2 k}-2^{k-1}-1=2^{a-1}$. Clearly, this equation is impossible since $a, k \geq 2$.

(3) $\mathscr{G}$ is of type $\mathrm{B}_{\ell}, \ell \geq 3$. Then $p$ is odd. By Table 2 and Appendix A.2 in [17], $S$ has an irreducible $p$-Brauer character of degree $2 \ell^{2}+\ell$ with trivial action of the center of $G(q)$. Hence $2 \ell^{2}+\ell$ must be a prime power, which is impossible since $\operatorname{gcd}(\ell, 2 \ell+1)=1$ and $\ell \geq 3$.

(4) $\mathscr{G}$ is of type $\mathrm{D}_{\ell}, \ell \geq 4$. Assume that $p=2$. Then the center of $G(q)$ is trivial. By [17, Table 2], $S$ has irreducible $p$-Brauer characters of degree $2 \ell$ and $2 \ell^{2}-\ell-\operatorname{gcd}(2, \ell)$. Arguing as in case $(2)$, we can see that these degrees cannot be prime power. So, this case cannot happen. Assume next that $p$ is odd. By Appendix A.2 and Table 2 in [17], the representation $L\left(\omega_{\ell-1}\right)$ is an irreducible module for $S$ with dimension $2 \ell^{2}-\ell$. But this degree cannot be a prime power since $\operatorname{gcd}(\ell, 2 \ell-1)=1$ and $\ell \geq 4$.

(5) $\mathscr{G}$ is of type $\mathrm{E}_{6}$. By Appendix A.2 and Table 2 in [17], the representation $L\left(\omega_{2}\right)$ is an irreducible module for $S$ with dimension 78 if $p \neq 3$ and 77 if $p=3$. Clearly, these degrees are not prime powers.

(6) $\mathscr{G}$ is of type $\mathrm{E}_{7}$. In this case, the representation $L\left(\omega_{1}\right)$ is an irreducible module for $S$ with dimension 133 if $p \neq 2$ and 132 if $p=3$ and these are not prime powers.

(7) For the remaining groups of type $\mathrm{G}_{2}, \mathrm{~F}_{4}$ and $\mathrm{E}_{8}$, the center of $G(q)$ is trivial. For groups of type $\mathrm{G}_{2}$, if $p \neq 3$, then $L\left(\omega_{1}\right)$ has dimension 14 and if $p=3$, then $L\left(2 \omega_{1}+\omega_{2}\right)$ has dimension 189 . For groups of type $\mathrm{F}_{4}$, the representation $L\left(\omega_{1}\right)$ has dimension 52 if $p \neq 2$ and 26 if $p=2$. Finally, for groups of type $\mathrm{E}_{8}$, the representation $L\left(\omega_{5}\right)$ has dimension 248 . We can easily check that these degrees are not prime powers. The proof is now complete.

We now consider the case when $S$ is a simple group of Lie type and $p$ is different from the defining characteristic of $S$.

Lemma 3.3: Let $S$ be a nonabelian simple group of Lie type defined over a field of size $q$ with $q=r^{f}$ and $S \neq{ }^{2} \mathrm{~F}_{4}(2)^{\prime}$. Let $p$ be a prime different from 
$r$. Suppose that $\sigma_{p}(S)=1$ and that $S$ is isomorphic to neither an alternating group nor $\mathrm{PSL}_{2}(8)$. Then the following hold:

(1) $S \cong \mathrm{PSL}_{2}(q)$ with $q \geq 17$ a Fermat prime and $p=2$,

(2) $S \cong \mathrm{PSL}_{2}\left(2^{f}\right)$ where $f \geq 5$ is a prime and $p=2^{f}-1$ is a Mersenne prime,

(3) $S \cong \mathrm{PSL}_{2}(7)$ and $p=2$.

Proof. Since $S$ is not isomorphic to $\mathrm{PSL}_{2}(q)$ for any $q \in\{4,5,8\}$, by Lemma 3.1(1) we can assume that $p$ divides $|S|$. By [4, Corollary 2], we deduce that $S$ has a complex irreducible character of $p$-defect zero and thus by Lemma 3.1(2), $p$ is an isolated degree in the defect zero graph $\Delta^{\circ}(S)$ of $S$. By [11, Theorem 3.10], we know that $|\pi(S)| \geq 3$. Now assume that $\Delta^{\circ}(S)$ has exactly two connected components. By Lemma 2.1, since $p$ is not the defining prime of $S$ and $|\pi(S)| \geq 3$, we deduce that $p$ must lie in a connected component of $\Delta^{\circ}(S)$ consisting of at least 2 vertices, a contradiction. Thus $\Delta^{\circ}(S)$ has three connected components and so by Lemma 2.1 again, one of the following cases holds:

(1) $S \cong \mathrm{PSL}_{2}(q)$ with $q=r^{f} \geq 4$. For this case, the degrees of the irreducible $p$-Brauer characters of $S$ are available in [7]. If $S \cong \operatorname{PSL}_{2}(7) \cong \operatorname{PSL}_{3}(2)$, then by using [5] we can check that all irreducible $p$-Brauer characters of $\mathrm{PSL}_{2}(7)$ have prime power degrees for some prime $p \neq 7$ if and only if $p=2$. This gives rise to case (3). Since $\mathrm{PSL}_{2}(5) \cong \mathrm{A}_{5}$ and $\mathrm{PSL}_{2}(9) \cong \mathrm{A}_{6}$, we can assume that $q \geq 11$. By [7, Table 2], we have that $q-1 \in \operatorname{cd}_{p}(S)$ and thus $q-1$ must be a prime power.

Assume that $q$ is odd. Then $q-1=2^{a}$ for some integer $a \geq 4$ since $q-1 \geq 10$ is even. Since $q=r^{f} \geq 11$, by Lemma 2.3 we have that $a=2^{m}$ for some integer $m \geq 2$ and $f=1$ so $q$ is a Fermat prime and $q \geq 17$. In particular, $q \equiv 1$ $(\bmod 4)$. Assume $p=2$. Since $((q-1) / 4)_{2^{\prime}}=1$, we obtain that $\operatorname{cd}_{2}(S)=$ $\{1,(q-1) / 2, q-1\}$. This proves (1). Assume $p \neq 2$. Then $(q+1) / 2 \in \operatorname{cd}_{p}(S)$ and so $(q+1) / 2=2^{2^{m}-1}+1$ must be a prime power. Since $2^{m}-1$ is odd, $2^{2^{m}-1}+1$ is always divisible by 3 , so $2^{2^{m}-1}+1=3^{k}$ for some integer $k \geq 1$. However, by Lemma 2.3, this equation holds only when $k=2$ and $a=4$ which implies that $q=1+2^{4}=17$. But then by using [5] we can check that $\mathrm{PSL}_{2}(17)$ does not satisfy the hypothesis of the lemma for any odd prime $p$.

Now assume that $q=2^{f}$ with $f \geq 4$. Hence $2^{f}-1=s^{b}$ for some prime $s$ and integer $b \geq 1$. By Lemma 2.3, we deduce that $f \geq 5$ is a prime and $b=1$. So, $q-1=s$ is a Mersenne prime. Now if $p=s$, then $\operatorname{cd}_{p}(S)=\{1, q-1, q\}$ since 
$p=q-1 \nmid q+1$. Hence, all irreducible $p$-Brauer characters of $S$ have prime power degrees. This is case (2) of the lemma. Assume now that $p \neq q-1$. Then $p \mid q+1$ and $(q-1)_{p^{\prime}} \neq 1$, so $\operatorname{cd}_{p}(S)=\{1, q-1, q+1\}$. Therefore, $q+1=u^{k}$ for some prime $u$ and integer $k \geq 1$. Then $2^{f}+1=u^{k}$ which implies that $u=3$ as $f$ is odd so that $2^{f}+1$ is divisible by 3 . Hence, we obtain that $f=3$ by Lemma 2.3, which is a contradiction.

(2) $S \cong \operatorname{PSL}_{3}(q), q=2^{6 m+1}-1$ with $6 m+1$ prime, or $q=3$. Since $S$ has an irreducible complex character of prime power degree $|S|_{p}$ by Lemma 3.1(2), where $p$ is not the defining characteristic of $S$, we deduce from [19, Theorem 1.1] that $\operatorname{gcd}(3, q-1)=1$ and $\left(q^{3}-1\right) /(q-1)$ is a prime power. The case when $q=3$ can be ruled out by using [5]. Hence, we can assume that $q=2^{6 m+1}-1$ with $6 m+1$ prime. However, we can see that $q-1=2\left(\left(2^{6}\right)^{m}-1\right)$ is divisible by $2^{6}-1=3^{2} \cdot 7$ and so by 3 . Thus $\operatorname{gcd}(3, q-1)=3>1$, which is a contradiction.

(3) $S \cong \mathrm{PSU}_{3}(q), q=2^{2^{2 m}}+1$ with $m \geq 0$, or $q=9$. As in the previous case, by applying [19, Theorem 1.1] we must have that $\operatorname{gcd}(3, q+1)=1$ and $\left(q^{3}+1\right) /(q+1)$ is a prime power. The cases when $q=9$ or $m=0$ can be ruled out by using [5]. For the remaining cases, we see that $q+1=2\left(2^{2^{2 m}}-1+1\right)$. Obviously, $2^{2 m}-1=4^{m}-1=3 k$, where $k \geq 1$ is odd. Then $q+1=$ $2\left(2^{3 k}+1\right)=2\left(8^{k}+1\right)$ is always divisible by $8+1=3^{2}$ since $k$ is odd, hence $\operatorname{gcd}(3, q+1)=3>1$, a contradiction.

(4) $S \cong \operatorname{PSp}_{4}(q)$ with $q \in\{3,5\}$. These cases can be ruled out by using [5]. The proof is now complete.

We are now ready to classify all simple groups whose all irreducible $p$-Brauer characters have prime power degrees.

Theorem 3.4: Let $S$ be a nonabelian simple group and let $p$ be a prime. If $\sigma_{p}(S)=1$, then one of the following cases holds:

(1) $S \cong \mathrm{A}_{5}$ or $\mathrm{PSL}_{2}(8)$ and $p$ is any prime,

(2) $S \cong \operatorname{PSL}_{2}(p)$ with $p$ odd and $7 \leq p \leq 13$,

(3) $S \cong \mathrm{PSL}_{2}\left(2^{f}\right)$ with $f \geq 4, \mathrm{PSp}_{4}\left(2^{f}\right)$ with $f \geq 2,{ }^{2} \mathrm{~B}_{2}\left(2^{2 f+1}\right)$ with $f \geq 1$, or $\mathrm{PSL}_{3}\left(2^{f}\right)$ with $1 \leq f \leq 2$; and $p=2$,

(4) $S \cong \mathrm{PSL}_{2}(q)$ with $q \geq 17$ a Fermat prime and $p=2$,

(5) $S \cong \mathrm{PSL}_{2}\left(2^{f}\right)$ where $f \geq 5$ is a prime and $p=2^{f}-1$ is a Mersenne prime,

(6) $S \cong \mathrm{A}_{6}$ and $p \in\{2,3\}$. 
Proof. If $S \cong \mathrm{A}_{5}$ or $\mathrm{PSL}_{2}(8)$, then all irreducible $p$-Brauer characters of $S$ have prime power degree for every prime $p$ by using [5]. Hence, we can assume from now on that $S$ is not isomorphic to one of these simple groups. By Lemma 3.1(1), we can also assume that $p$ divides $|S|$. We will use the classification of finite simple groups.

Assume first that $S$ is a sporadic simple group or the Tits group. Assume that $S$ has a complex irreducible character of $p$-defect zero. By Lemma 3.1(2), $S$ has a complex irreducible character $\chi \in \operatorname{Irr}(S)$ with $\chi(1)=|S|_{p}$. By [19, Theorem 1.1], we have that $S \cong \mathrm{M}_{11}$ and $p \in\{2,11\}$ or $S \cong \mathrm{M}_{12}$ and $p=11$, or $S \in\left\{\mathrm{M}_{24}, \mathrm{Co}_{2}, \mathrm{Co}_{3}\right\}$ and $p=23$. As the $p$-Brauer character tables of these simple groups are available in [5], it is routine to check that all the pairs $(S, p)$ above do not satisfy the hypothesis of the theorem. Assume now that $S$ has no complex irreducible character of $p$-defect zero. Then $S$ and $p$ appear in $[4$, Corollary 2]. For these cases, the $p$-Brauer character tables of $S$ are available in GAP [5] except for the cases $S \in\left\{\mathrm{Co}_{1}, \mathrm{~B}\right\}$ with $p=2$. Now using [5] and [14] for the exceptions above, we can easily check that these cases give rise to no examples of simple groups satisfying the hypothesis of the theorem.

Now if $S$ is an alternating group of degree at least 6 , then the result follows from [1, Corollary 1.3]. This gives rise to case (6). Finally, for simple group of Lie type which is not isomorphic to an alternating group, we obtain Cases $(2)-(5)$ by Lemmas 3.2 and 3.3. The proof is now complete.

Recall that if $G$ is a group and $p$ is a prime, then $\mathbf{O}_{p}(G)$ is the largest normal $p$-subgroup of $G$. Moreover, $\mathbf{O}_{p}(G)$ is contained in the kernel of every irreducible $p$-Brauer character of $G$, hence $\operatorname{cd}_{p}\left(G / \mathbf{O}_{p}(G)\right)=\operatorname{cd}_{p}(G)$. Now assume that $G$ is a quasisimple groups whose all irreducible $p$-Brauer characters have prime power degrees for some prime $p$. Since $\operatorname{cd}_{p}(G / \mathrm{Z}(G)) \subseteq \operatorname{cd}_{p}(G)$, we see that $G / \mathrm{Z}(G)$ satisfies the hypotheses of Theorem 3.4, so $G / \mathrm{Z}(G)$ appears in the conclusion of that theorem. As the Schur multiplier of these simple groups are known, using $[5,7,17]$, we can easily deduce the following.

Corollary 3.5: Let $G$ be a quasisimple group and let $p$ be a prime. If $\sigma_{p}(G)=$ 1 and $\mathrm{Z}(G)$ is nontrivial, then the following cases hold:

(1) $G / \mathrm{Z}(G) \cong \mathrm{A}_{5}$ and $p \in\{2,5\}$,

(2) $G / \mathrm{Z}(G) \cong{ }^{2} \mathrm{~B}_{2}(8), \mathrm{PSL}_{3}(2)$ or $\mathrm{PSL}_{3}(4) ; p=2$ and $\mathrm{Z}(G)$ is a 2-group,

(3) $G / \mathrm{Z}(G) \cong \operatorname{PSL}_{2}(q)$ with $q \geq 17$ a Fermat prime and $p=2$, 
(4) $G / \mathrm{Z}(G) \cong \mathrm{A}_{6}$ and $p=2$ or $\mathrm{Z}(G)$ is a 3 -group and $p=3$.

Proof. As the center $\mathrm{Z}(G)$ of $G$ is nontrivial, by applying Theorem 3.4 one of the following cases holds:

(1) $G \cong 2 \cdot \mathrm{A}_{5}$. Using [5], we have that $p \in\{2,5\}$. For other primes, we can see that $6 \in \operatorname{cd}_{p}(G)$.

(2) $G \cong \mathrm{SL}_{2}(p)$ with $7 \leq p \leq 13$. By [17, Remark 4.5], we have that $6 \in$ $\operatorname{cd}_{p}(G)$, so $G$ does not satisfy the hypothesis of the corollary.

(3) $G / \mathrm{Z}(G) \cong \mathrm{PSL}_{3}\left(2^{f}\right)$ with $1 \leq f \leq 2$ or ${ }^{2} \mathrm{~B}_{2}(8)$ and $p=2$. Recall that the Schur multiplier of $\mathrm{PSL}_{3}(2), \mathrm{PSL}_{3}(4)$ and ${ }^{2} \mathrm{~B}_{2}(8)$ is isomorphic to $\mathbb{Z}_{2}, \mathbb{Z}_{3} \times \mathbb{Z}_{4} \times$ $\mathbb{Z}_{4}$ or $\mathbb{Z}_{2}^{2}$, respectively. Using [5], we can check that $G$ satisfies the hypothesis of the corollary only when $\mathrm{Z}(G)$ is a 2 -group. Indeed, $\operatorname{cd}_{2}(G)$ always contains 24 if 3 divides $|\mathrm{Z}(G)|$.

(4) $G \cong \mathrm{SL}_{2}(q)$ with $q \geq 17$ a Fermat prime and $p=2$. Since $\mathrm{Z}(G)$ is a $p$-group, $G$ satisfies the hypothesis of the corollary.

(5) $G / \mathrm{Z}(G) \cong \mathrm{A}_{6}$ and $p \in\{2,3\}$. In this case, the Schur multiplier of $\mathrm{A}_{6}$ is cyclic of order 6 . If $p=2$, then $G$ always satisfies the hypothesis of the corollary for all possibilities of $\mathrm{Z}(G)$. When $p=3, G$ satisfies the hypothesis of the corollary only when $\mathrm{Z}(G)$ is a 3 -group.

Theorem A now follows from Theorem 3.4 and Corollary 3.5. The next result will be needed in the proof of Theorem $\mathrm{C}$.

Corollary 3.6: Let $S$ be a nonabelian simple group and let $p$ be a prime. If all nonlinear irreducible $p$-Brauer characters of $S$ have prime degrees, then $S \cong \mathrm{PSL}_{2}(p)$ with $p \in\{5,7\}$.

When $p$ is an odd prime, we obtain the following.

Corollary 3.7: Let $G$ be a quasisimple group and let $p$ be an odd prime. If $\sigma_{p}(G)=1$, then the following hold:

(1) $G \cong \operatorname{PSL}_{2}\left(2^{f}\right)$ where $f \geq 5$ is a prime and $p=2^{f}-1$ is a Mersenne prime,

(2) $G \cong \mathrm{A}_{5}$ or $\mathrm{PSL}_{2}(8)$ and $p$ is any odd prime,

(3) $G \cong \mathrm{PSL}_{2}\left(p^{f}\right)$ with $p$ odd and $7 \leq p^{f} \leq 13$,

(4) $G \cong 2 \cdot \mathrm{A}_{5}$ and $p=5$,

(5) $G \cong 3 \cdot \mathrm{A}_{6}$ and $p=3$. 
Moreover, $G$ has at least two nontrivial $p$-Brauer character degrees $u$ and $v$, where $u$ is a power of $p$ and $v$ is a power of a prime $s$ different from $p$.

Recall that a group $G$ is said to be an almost simple group with socle $S$ if $S$ is a nonabelian simple group and $S \unlhd G \leq \operatorname{Aut}(S)$.

Corollary 3.8: Let $G$ be an almost simple group with simple socle $S$ and let $p$ be an odd prime. Suppose that $\sigma_{p}(G)=1$ and $G \neq S$. Then the following hold:

(1) $G \cong \mathrm{PSL}_{2}(8) \cdot 3$ and $p=3$ or

(2) $G \cong \mathrm{PGL}_{2}\left(p^{f}\right)$ with $5 \leq p^{f} \leq 13$.

Proof. Since $S \unlhd G$ and $\sigma_{p}(G)=1$, we deduce that $\sigma_{p}(S)=1$, so $S$ is one of the simple groups given in Cases (1) - (3) of Corollary 3.7. The last two cases can be deal with easily by using [5]. Hence, we can assume that $S \cong \mathrm{PSL}_{2}\left(2^{f}\right)$ with $f \geq 5$ a prime and $p=2^{f}-1$ a Mersenne prime. In this case, we have that $\mathrm{SL}_{2}\left(2^{f}\right) \cong \mathrm{PGL}_{2}\left(2^{f}\right) \cong \mathrm{PSL}_{2}\left(2^{f}\right)$ and $\operatorname{Aut}(S) \cong S\langle\varphi\rangle$, where $\varphi$ is a field automorphism of $S$ of prime order $f$. It follows that $G=S\langle\varphi\rangle$ as $|G: S|>1$. Since $f \geq 5,2^{f}+1$ has a primitive prime divisor $r>3$. Let $\mathscr{G}$ be a simply connected simple algebraic group and $F$ be a Frobenius map of $\mathscr{G}$ such that $\mathscr{G}^{F} \cong \mathrm{SL}_{2}\left(2^{f}\right)$. Let $\left(\mathscr{G}^{*}, F^{*}\right)$ be dual to $(\mathscr{G}, F)$. Then $\left(\mathscr{G}^{*}\right)^{F^{*}} \cong$ $\mathrm{PGL}_{2}\left(2^{f}\right) \cong S$. Let $s \in \mathrm{PGL}_{2}\left(2^{f}\right) \cong S$ be a semisimple element of order $r$. We have that $\left|\mathrm{C}_{S}(s)\right|=2^{f}+1$. Let $\varphi^{*}$ be an automorphism of $\mathscr{G}^{*}$ induced by the field automorphism $x \mapsto x^{2}$. Then $r$ does not divide $\left|\left(\mathscr{G}^{*}\right)^{\varphi^{*}}\right|$. By [3, Lemma 2.5], $S \cong \mathrm{SL}_{2}\left(2^{f}\right)$ has an irreducible character $\chi_{s}$ of order $\left|S: \mathrm{C}_{S}(s)\right|_{2^{\prime}}=2^{f}-1$ and $\chi_{s}$ is not $\varphi$-invariant. Since $\chi_{s}(1)=p$, both $\chi_{s}$ and $\chi_{s}^{\varphi}$ are of $p$-defect zero and so $\chi_{s}^{\varphi}(x)=0=\chi_{s}(x)$ for any $x \in S$ with $|x|=p$. Notice that all nontrivial $p$-singular elements of $S$ have order $p$. As $\chi_{s}$ is not $\varphi$-invariant, it follows that $\chi_{s}^{\varphi}(y) \neq \chi_{s}(y)$ for some $p$-regular element $y \in S$. In particular, $\lambda=\chi_{s}^{\circ} \in \operatorname{IBr}_{p}(S)$ is not $\varphi$-invariant and thus we deduce that $\lambda^{G} \in \operatorname{IBr}_{p}(G)$, so $\lambda^{G}(1)=p f \in \operatorname{cd}_{p}(G)$, which is impossible.

\section{Groups whose all irreducible $p$-Brauer characters have prime power degrees}

We will prove Theorems B and $\mathrm{C}$ in this section. Recall that the prime graph $\Delta(G)$ of a group $G$ is a graph whose vertex set is $\rho(G)$ and there is an edge 
between two distinct primes $r$ and $s$ if and only if the product $r s$ divides some complex character degree of $G$. The structure of a group $G$ whose prime graph $\Delta(G)$ is disconnected has been studied in [16]. We first begin with the following technical lemma.

Lemma 4.1: Let $p$ be an odd prime and let $N$ be a normal subgroup of a perfect group $G$ such that $G / N$ is a quasisimple group and $\mathbf{O}_{p}(G)=1$. Suppose that no nontrivial irreducible $p$-Brauer characters of $N$ is $G$-invariant and $\sigma_{p}(G)=1$. Then $N$ is trivial.

Proof. Suppose by contradiction that $N$ is nontrivial.

Claim 1. If $1_{N^{\circ}} \neq \lambda \in \operatorname{IBr}_{p}(N)$ and $I=I_{G}(\lambda)$, then the following hold:

(1) $G / N \cong \mathrm{A}_{5}, I / N \cong \mathrm{A}_{4},|G: I|=5$ and $p$ is any odd prime,

(2) $G / N \cong 2 \cdot \mathrm{A}_{5}, I / N \cong \mathrm{SL}_{2}(3),|G: I|=5$ and $p=5$,

(3) $G / N \cong \mathrm{PSL}_{2}(8), I / N \cong 2^{3}: 7,|G: I|=3^{2}$ and $p$ is any odd prime,

(4) $G / N \cong \mathrm{PSL}_{2}(11), I / N \cong \mathrm{A}_{5},|G: I|=11$ and $p=11$,

(5) $G / N \cong \mathrm{PSL}_{2}(7), I / N \cong 7: 3$ or $\mathrm{S}_{4},|G: I|=8$ or 7 , respectively and $p=7$.

Furthermore, $I / N$ is a nonabelian Hall subgroup of $G / N$.

Let $\lambda \in \operatorname{IBr}_{p}(N)$ be a nontrivial $p$-Brauer character of $N$. By our assumption, we have that $N \unlhd I:=I_{G}(\lambda) \lessgtr G$. By [24, Theorem 8.9], we have that $\varphi^{G} \in$ $\operatorname{IBr}_{p}(G)$ for any $\varphi \in \operatorname{IBr}_{p}(I \mid \lambda)$, hence $\varphi^{G}(1)=|G: I| \varphi(1) \in \operatorname{cd}_{p}(G)$ is a prime power and so $|G: I|$ is a prime power. Since $\sigma_{p}(G / N)=1$, we deduce that $G / N$ is one of the quasisimple groups in Corollary 3.7. Now the result follows by applying [6, Theorem 1] and Lemma 2.3. The last statement is obvious.

Claim 2. For every $1_{N^{\circ}} \neq \lambda \in \operatorname{IBr}_{p}(N)$, we have that $p|| I / N \mid$ with $I=I_{G}(\lambda)$, and one of the following cases holds:

(i) $G / N \cong \mathrm{A}_{5}, I / N \cong \mathrm{A}_{4},|G: I|=5$ and $p=3$, or

(ii) $G / N \cong \mathrm{PSL}_{2}(8), I / N \cong 2^{3}: 7,|G: I|=3^{2}$ and $p=7$, or

(iii) $G / N \cong \mathrm{PSL}_{2}(7), I / N \cong 7: 3,|G: I|=8$, and $p=7$.

By way of contradiction, suppose that $p \nmid|I / N|$. By Claim 1, one of the following cases holds:

(a) $G / N \cong \mathrm{A}_{5}, I / N \cong \mathrm{A}_{4},|G: I|=5$ and $p \geq 5$,

(b) $G / N \cong 2 \cdot \mathrm{A}_{5}, I / N \cong \mathrm{SL}_{2}(3),|G: I|=5$ and $p=5$,

(c) $G / N \cong \mathrm{PSL}_{2}(8), I / N \cong 2^{3}: 7,|G: I|=3^{2}$ and $3 \leq p \neq 7$

(d) $G / N \cong \mathrm{PSL}_{2}(11), I / N \cong \mathrm{A}_{5},|G: I|=11$ and $p=11$, 
(e) $G / N \cong \mathrm{PSL}_{2}(7), I / N \cong \mathrm{S}_{4},|G: I|=7$, and $p=7$.

Observe that in all cases, $I / N$ is a nonabelian $p^{\prime}$-group and $\operatorname{gcd}(|G: I|,|I / N|)=$ 1. Assume first that $\lambda$ extends to $\lambda_{0} \in \operatorname{IBr}_{p}(I)$. By [24, Corollary 8.20], $\lambda_{0} \mu \in$ $\operatorname{IBr}_{p}(I \mid \lambda)$ for all $\mu \in \operatorname{IBr}_{p}(I / N)$. Since $\operatorname{IBr}_{p}(I / N)=\operatorname{Irr}(I / N)$ and $I / N$ is nonabelian, $I / N$ has a nonlinear irreducible $p$-Brauer character $\mu$ such that $p \nmid \mu(1)$. It follows that $\left(\lambda_{0} \mu\right)^{G} \in \operatorname{IBr}_{p}(G)$ with degree $|G: I| \lambda(1) \mu(1)$. Obviously, this degree cannot be a prime power. Thus $\lambda$ is $I$-invariant but not extendible to $I$. Hence, there exists $\varphi \in \operatorname{IBr}_{p}(I \mid \lambda)$ with $\varphi_{N}=e \lambda$, where $e$ is nontrivial. Since $|I / N|$ is prime to $p, I / N$ is $p$-solvable and so by [24, Theorem 8.30], we obtain that $\varphi(1) / \lambda(1)=e$ divides $|I / N|$. In particular, $\operatorname{gcd}(e,|G: I|)=1$. Since $\varphi^{G} \in \operatorname{IBr}_{p}(G)$, we deduce that $\varphi^{G}(1)=|G: I| e \lambda(1)$ is a prime power, which is impossible. Therefore, we conclude that $p$ divides $|I / N|$. Now the result follows by checking the list given in the previous claim.

Claim 3. $N$ is a solvable $p^{\prime}$-group.

By Claim 2, for every $1_{N^{\circ}} \neq \lambda \in \operatorname{IBr}_{p}(N)$, we have that $|G: I|=r^{a}$ for some fixed prime $r \neq p$ and some integer $a \geq 1$. Hence, if $\varphi \in \operatorname{IBr}_{p}(I \mid \lambda)$, then $\varphi^{G}(1)=r^{a} \varphi(1) \in \operatorname{cd}_{p}(G)$ must be an $r$-power. Since $\lambda(1) \mid \varphi(1)$, we deduce that $\lambda(1)$ is an $r$-power, thus the degree of every irreducible $p$-Brauer character of $N$ is a power of $r$, so by [26, Corollary 1.2], $N$ is solvable as $p$ is odd. Moreover, by [23, Theorem 5.5] the Sylow $p$-subgroup $P$ of $N$ is normal in $N$. Since $N \unlhd G$, we must have that $P \unlhd G$ and thus $P \subseteq \mathbf{O}_{p}(G)=1$, hence $P=1$ which implies that $N$ is a solvable $p^{\prime}$-group as wanted.

The final contradiction Let $L \unlhd N$ be a normal subgroup of $G$ such that $N / L$ is a chief factor of $G$. Since $N$ is a solvable $p^{\prime}$-subgroup of $G$, we deduce that $N / L$ is an elementary abelian $s$-group for some prime $s$ different from $p$. Without lost of generality, we can assume that $L=1$. Then $N$ is a minimal normal subgroup of $G$, where $G / N \cong \operatorname{PSL}_{2}(q)$ with $q \in\{5,7,8\}$ and $G$ is perfect.

Since $|N|$ is prime to $p$, we have that $\operatorname{Irr}(N)=\operatorname{IBr}_{p}(N)$. For every $1_{N} \neq \theta \in$ $\operatorname{Irr}(N), \theta$ can be considered as a $p$-Brauer character of $N$ and hence $I / N \cong$ $\mathrm{A}_{4}, 2^{3}: 7$ or $7: 3$ when $q=5,8$ or 7 , respectively, where $I=I_{G}(\theta)$. Notice that $p=3,7$ or 7 , respectively. Then by [24, Theorem 8.15$], \theta$ determines a unique element $[\theta]_{I / N}$ of the Schur Multiplier $\mathbb{M}\left(I / N, \mathbb{F}^{*}\right)=\mathbb{H}^{2}\left(I / N, \mathbb{F}^{*}\right)$, where $\mathbb{F}$ is an algebraically closed field of characteristic $p$. It is also well known that $\mathbb{M}\left(I / N, \mathbb{F}^{*}\right)$ is isomorphic to the $p^{\prime}$-part of the Schur multiplier $\mathbb{M}(I / N):=$ 
$\mathbb{M}\left(I / N, \mathbb{C}^{*}\right)$ in characteristic zero. Now if $I / N \cong 7: 3$, then $\mathbb{M}(I / N)$ is trivial since all Sylow $r$-subgroups of $I / N$ are cyclic. Assume that $I / N \cong 2^{3}: 7 \cong H$. Since $\mathbb{M}\left(\mathrm{PSL}_{2}(8)\right)=1$ and $H$ is the normalizer in $\mathrm{PSL}_{2}(8)$ of an abelian Sylow 2-subgroup, we obtain that $\mathbb{M}(H)_{2} \cong \mathbb{M}\left(\mathrm{PSL}_{2}(8)\right)_{2}=1$ (see [8]). Moreover, $\mathbb{M}(H)$ is a 2-group as the Sylow 7-subgroups of $H$ are cyclic, so $\mathbb{M}(H)=1$. Finally, if $I / H \cong \mathrm{A}_{4}$, then $\mathbb{M}(H) \cong \mathbb{Z}_{2}$.

Assume that $q \in\{5,8\}$. Let $1_{N^{\circ}} \neq \lambda \in \operatorname{IBr}_{p}(N)=\operatorname{Irr}(N)$. Observe that the Sylow $p$-subgroup of $I / N$ is cyclic of order $p$ and it is not normal in $I / N$, where $I=I_{G}(\lambda)$. By [5], we see that $I / N$ has an irreducible $p$-Brauer character $\mu$ of degree $p$. If $\lambda$ extends to $\lambda_{0} \in \operatorname{IBr}_{p}(I)$, then $\lambda_{0} \mu \in \operatorname{IBr}_{p}(I \mid \lambda)$ and thus $\left(\lambda_{0} \mu\right)^{G} \in \operatorname{IBr}_{p}(G)$ so that $\left(\lambda_{0}\right) \mu^{G}(1)=|G: I| \mu(1)=p|G: I|$ is a prime power which is impossible as $p \nmid|G: I|$. Therefore, $\lambda$ is not extendible to $I$. It follows that $G / N \cong \mathrm{A}_{5}$ and $I / N \cong \mathrm{A}_{4}$ since $\mathbb{M}\left(2^{3}: 7, \mathbb{F}^{*}\right)=1$. Since $\mathbb{M}\left(\mathrm{A}_{4}\right) \cong \mathbb{Z}_{2}$ and the full covering group of $\mathrm{A}_{4}$ is $2 \cdot \mathrm{A}_{4} \cong \mathrm{SL}_{2}(3)$, using the theory of modular character triple isomorphisms [24, Theorem 8.28], there exists $\varphi \in \operatorname{IBr}_{p}(I \mid \lambda)$ such that $\varphi_{N}=2 \lambda$ as $\mathrm{SL}_{2}(3)$ has a faithful irreducible $p$-Brauer character of degree 2. Hence, $\varphi^{G} \in \operatorname{IBr}_{p}(G)$ and so $\varphi^{G}(1)=5 \cdot 2=10$, which is not a prime power, a contradiction.

Assume that $q=7$. As the Schur multiplier of $I / N \cong 7: 3$ is trivial, $\theta$ extends to $\theta_{0} \in \operatorname{Irr}\left(I_{G}(\theta) \mid \theta\right)$. By Gallagher's theorem [11, Corollary 6.17], $\theta_{0} \mu$ with $\mu \in$ $\operatorname{Irr}\left(I_{G}(\theta) \mid\right)$ are all the irreducible constituents of $\theta^{I}$. Since $\operatorname{cd}\left(I_{G}(\theta) / N\right)=\{1,3\}$, we deduce that every irreducible character in $\operatorname{Irr}(G \mid \theta)$ has degree 8 or 24 . Since this is true for all $\theta \in \operatorname{Irr}(N)$, we deduce that $\operatorname{cd}(G)=\{8,24\} \cup \operatorname{cd}(G / N)=$ $\{1,3,6,7,8,24\}$. It follows that the prime graph $\Delta(G)$ has exactly two connected components. Since $G$ is not simple, by [16, Theorem 6.2] either $G \cong \mathrm{SL}_{2}\left(q_{1}\right)$ for some odd prime power $q_{1} \geq 5$ or $G$ has a normal subgroup $L$ such that $G / L \cong \mathrm{SL}_{2}\left(q_{1}\right)$ for some prime power $q_{1} \geq 4, L$ is elementary abelian of order $q_{1}^{2}$, and $G / L$ acts transitively on the nontrivial characters in $\operatorname{Irr}(L)$. As $G / N \cong$ $\mathrm{PSL}_{2}(7)$, in both cases, we deduce that $q_{1}=7$ and $G$ has a quotient group which is isomorphic to $\mathrm{SL}_{2}(7)$. However, this is impossible as $\sigma_{7}\left(\mathrm{SL}_{2}(7)\right)>1$. The proof is now complete.

Theorem 4.2: Let $p$ be an odd prime and let $G$ be a perfect group. Suppose that $\sigma_{p}(G)=1$ and $\mathbf{O}_{p}(G)=1$. Then $G$ is a quasisimple group and the possibilities for $G$ and $p$ are given in Cases (1) - (4) in Corollary 3.7. 
Proof. Let $N$ be a maximal normal subgroup of $G$. Since $G$ is perfect, $G / N$ is a nonabelian simple group and $\sigma_{p}(G / N)=1$. Hence, $G / N$ is isomorphic to one of the simple groups in Cases (1) - (3) of Corollary 3.7.

(1) Assume that there exists a nontrivial $p$-Brauer character $\lambda \in \operatorname{IBr}_{p}(N)$ such that $\lambda$ is $G$-invariant. If $\lambda$ extends to $\lambda_{0} \in \operatorname{IBr}_{p}(G)$, then $\lambda_{0}(1)=\lambda(1) \geq$ 2 since $G$ is perfect. By [24, Corollary 8.20], $\lambda_{0} \mu$ for $\mu \in \operatorname{IBr}_{p}(G / N)$ are all the irreducible constituents of $\lambda^{G}$. By Corollary 3.7, $G / N$ has two irreducible $p$-Brauer characters $\mu_{i}, i=1,2$ such that $\mu_{i}(1)$ are prime powers and $\operatorname{gcd}\left(\mu_{1}(1), \mu_{2}(1)\right)=1$. But then for $i=1,2, \lambda_{0}(1) \mu_{i}(1) \in \operatorname{cd}_{p}(G)$ cannot be prime powers as $\lambda_{0}(1) \geq 2$. Therefore, we conclude that $\lambda$ is $G$-invariant but not extendible to $G$. By the theory of modular character triple isomorphisms [24, Theorem 8.28], the triple $(G, N, \lambda)$ is modular character triple isomorphic to the triple $(\Gamma, A, \mu)$, where $\Gamma$ is perfect, $\Gamma / A \cong G / N, \mu$ is a faithful linear character of $A$ and $A$ is a central $p^{\prime}$-subgroup of $\Gamma$. It follows that $\Gamma \cong \mathrm{SL}_{2}\left(p^{f}\right)$ with $7 \leq p^{f} \leq 13$ and $p^{f}$ being odd or $\Gamma \cong \mathrm{SL}_{2}(5)$ and $p$ is any odd prime; moreover, $A=\mathrm{Z}(\Gamma) \cong \mathbb{Z}_{2}$. Now if $e>1$ is the degree of some faithful irreducible $p$-Brauer character of $\Gamma$, then $\varphi_{N}=e \lambda$ for some $\varphi \in \operatorname{IBr}_{p}(G \mid \lambda)$ and thus $e$ must be a prime power. As 6 is always a degree of some faithful irreducible $p$-Brauer character of $\mathrm{SL}_{2}\left(p^{f}\right)$ for odd $p^{f}>5$, or $\mathrm{SL}_{2}(5)$ when $3 \leq p \neq 5$, we must have that $G / N \cong \operatorname{PSL}_{2}(5)$ and $p=5$. As the degrees of all faithful irreducible 5 -Brauer characters of $\mathrm{SL}_{2}(5)$ are powers of 2 , we deduce that $\lambda(1)$ must be a 2-power whenever $\lambda \in \operatorname{IBr}_{p}(N)$ is $G$-invariant.

We next claim that all irreducible 5-Brauer characters of $N$ is $G$-invariant and thus all elements in $\operatorname{cd}_{5}(N)$ are powers of 2 hence $N$ is solvable by [26, Corollary 1.2], so by [23, Theorem 5.5] we deduce that the Sylow 5 -subgroup of $N$ is normal in $N$ and thus it is contained in $\mathbf{O}_{5}(G)=1$ so that $N$ must be a solvable $5^{\prime}$-group. To prove this claim, suppose that $N$ has an irreducible 5-Brauer character $\mu$ such that $\mu$ is not $G$-invariant and let $I=I_{G}(\mu)$. Let $\varphi \in \operatorname{IBr}_{5}(I \mid \mu)$. Then $\varphi^{G}(1)=|G: I| \varphi(1) \in \operatorname{cd}_{5}(G)$ is prime power, so $|G: I|$, the index of a subgroup of $\mathrm{PSL}_{2}(5)$, is a prime power. It follows that $I / N \cong \mathrm{A}_{4}$ and $|G: I|=5$. Now arguing as in the proof of Claim 2 of Lemma 4.1, we obtain a contradiction. Thus $N$ is a solvable $5^{\prime}$-group. Let $\theta \in \operatorname{Irr}(N)$ be a linear character of $N$. Then $\theta$ is also a 5-Brauer character of $N$ and so $\theta$ is $G$-invariant but not extendible to $G$ since $G$ is perfect. It follows that $G / \operatorname{ker}(\theta) \cong \mathrm{SL}_{2}(5)$. Now let $M=\operatorname{ker}(\theta) \unlhd G$. If a nontrivial $p$-Brauer character $\lambda \in \operatorname{IBr}_{5}(M)$ is $G$-invariant, then it must extend to $G$ as the Schur multiplier of $\mathrm{SL}_{2}(5)$ is 
trivial and so with the same reasoning as in the previous paragraph, we obtain a contradiction. Thus no nontrivial irreducible 5-Brauer character of $M$ is $G$ invariant and hence by applying Lemma $4.1, M$ is trivial, so $G \cong \mathrm{SL}_{2}(5)$ and $p=5$. This is Case (4) in Corollary 3.7.

(2) Assume now that no nontrivial irreducible $p$-Brauer character of $N$ is $G$-invariant. It follows from Lemma 4.1 that $N$ is trivial and so $G$ is one of the simple groups in Cases (1) - (3) of Corollary 3.7. The proof is now complete.

We are now ready to prove Theorem B.

Proof of Theorem B. Let $K$ be the last term of the derived series of $G$. Since $G$ is nonsolvable, we deduce that $K$ is a nontrivial perfect normal subgroup of $G$. Moreover, $\sigma_{p}(K)=1$ so by Theorem $4.2, K$ is quasisimple and the possibilities for $K$ and $p$ are given in Cases (1) - (4) of Corollary 3.7. We note that $Z:=\mathrm{Z}(K)$ is cyclic of order at most 2 and thus $Z \subseteq \mathrm{Z}(G)$. Let $C$ be a normal subgroup of $G$ defined by $C / Z=\mathrm{C}_{G / Z}(K / Z)$. Then $Z \unlhd C \unlhd G$ and $[K, C] \leq Z$. Since $Z=\mathrm{Z}(K)$, we have that $[K, C, K]=[C, K, K]=1$. By Three Subgroups Lemma, we deduce that $[K, K, C]=1$, which implies that $C$ centralizes $[K, K]=K$, hence $[C, K]=1$. In particular, $C=\mathrm{C}_{G}(K)$ and $G / C$ is an almost simple group with simple socle $K / Z$.

Claim 1. All irreducible $p$-Brauer characters of $C / Z$ is linear.

Let $L=C K \unlhd G$. Then $L / Z \cong C / Z \times K / Z$ since $K \cap C=Z$ and $[C, K]=1$. Since $\mathbf{O}_{p}(G)=1$, we obtain that $\mathbf{O}_{p}(C)=1$. As $\sigma_{p}(G)=1$, we deduce that $\sigma_{p}(K / Z)=\sigma_{p}(L / Z)=1$. By Corollary 3.7, there exist $u, v \in \operatorname{cd}_{p}(K / Z)$ such that $u, v$ are nontrivial prime powers and $\operatorname{gcd}(u, v)=1$. Let $\lambda \in \operatorname{IBr}_{p}(C / Z)$. Then both $\lambda(1) u$ and $\lambda(1) v$ lie in $\operatorname{cd}_{p}(L / Z)$ by [24, Theorem 8.21], so they are both prime powers, which is impossible unless $\lambda(1)=1$. Hence, all irreducible $p$-Brauer characters of $C / Z$ are linear.

Claim 2. $C / Z$ is an abelian $p^{\prime}$-group and if $Z$ is nontrivial, then all irreducible $p$-Brauer characters of $C$ have 2-power degrees.

Assume first that $Z=1$. Then $K$ is a nonabelian simple group and by Claim 1, all irreducible $p$-Brauer characters of $C$ are linear. Since $\mathbf{O}_{p}(C)=1$, it follows that $C$ is an abelian $p^{\prime}$-group.

Assume that $Z$ is nontrivial. Then $Z \cong \mathbb{Z}_{2}, K \cong \mathrm{SL}_{2}(5)$ and $p=5$. By Claim $1, \operatorname{IBr}_{p}(C / Z)$ contains only linear $p$-Brauer characters. Let $L=C K \unlhd G$. 
Now let $\lambda \in \operatorname{IBr}_{p}(C)$ with $\lambda \neq 1_{C^{\circ}}$. Then $\lambda_{Z}=\lambda(1) \mu$ for some nontrivial $p$ Brauer character $\mu \in \operatorname{IBr}_{p}(Z)=\operatorname{Irr}(Z)$. It follows that $\lambda \in \operatorname{IBr}_{p}(C \mid \mu)$. Using [5], $\operatorname{IBr}_{p}(K \mid \mu)$ contains an irreducible $p$-Brauer character $\gamma$ of degree 2. Since $L=C K,[C, K]=1$ and $L / Z \cong C / Z \times K / Z$, by applying [25, Lemma 2.2] we obtain that $\chi=\lambda \cdot \gamma \in \operatorname{IBr}_{p}(L \mid \mu)$ with $\chi(1)=\lambda(1) \gamma(1)=2 \lambda(1)$. As $\sigma_{p}(L)=1$, we must have that $\chi(1)=2 \lambda(1)$ is a 2 -power, so $\lambda$ must be a power of 2 . Therefore, we conclude that all irreducible $p$-Brauer characters of $C$ have 2power degrees. By [26, Corollary 1.2] and [23, Theorem 5.5] we have that $C$ is a solvable $p^{\prime}$-group since $p$ is odd and $\mathbf{O}_{p}(C)=1$. This forces $C / Z$ is a $p^{\prime}$-group and so $C / Z$ must be abelian.

Claim 3. $K=G^{\prime}$.

If $G / C$ is simple, then $G=C K$ and thus $G / Z \cong C / Z \times K / Z$. Since $C / Z$ is abelian by Claim 2, we deduce that $(G / Z)^{\prime}=K / Z$, which implies that $K=G^{\prime}$ since $Z \leq K$. Hence, we can assume that $G / C$ is not simple. Notice that $\sigma_{p}(G / C)=1$ so $G / C$ and $p$ are given in Corollary 3.8. In particular, $(G / C)^{\prime}$ is simple and either $(G / C)^{\prime} \cong \mathrm{PSL}_{2}(8)$ and $p=3$ or $(G / C)^{\prime} \cong \mathrm{PSL}_{2}\left(p^{f}\right)$ with $5 \leq p^{f} \leq 13$. We have that $(G / C)^{\prime}=G^{\prime} C / C=K C / C \cong K / Z$. In all cases, $K / Z$ has a nontrivial irreducible $p$-Brauer character $\lambda$ of degree 7 if $K / Z \cong \mathrm{PSL}_{2}(8)$ and $p^{f}$ otherwise, which is extendible to $G / C$. We also note that $\operatorname{gcd}(|G: C K|, \lambda(1))=1$. Since $C K / Z \cong C / Z \times K / Z$, we deduce that $\varphi=1_{C / Z} \times \lambda \in \operatorname{IBr}_{p}(C K / Z)$ is an extension of $\lambda$ to $C K$. As $G / C K$ is cyclic of order 3 or 2 , if $\varphi$ is not $G$-invariant, then $\varphi^{G} \in \operatorname{IBr}_{p}(G)$, so $\varphi^{G}(1)=\mid G$ : $C K \mid \lambda(1) \in \operatorname{cd}_{p}(G)$, which is impossible as this degree cannot be a prime power. Thus $\varphi$ is $G$-invariant and so by [24, Theorem 8.12], $\varphi$ extends to $\lambda_{0} \in \operatorname{IBr}_{p}(G)$, therefore $\lambda$ is extendible to $\lambda_{0} \in \operatorname{IBr}_{p}(G)$. Now by applying [24, Corollary 8.20], $\lambda_{0}(1) \mu(1) \in \operatorname{cd}_{p}(G)$ for all $\mu \in \operatorname{IBr}_{p}(G / K)$. Observe that $G / K$ has a normal abelian $p^{\prime}$-subgroup $C K / K$ with index $|G: C K|=3$ or 2 . It follows that $\operatorname{cd}_{p}(G / K) \subseteq\{1,2,3\}$. Since $\lambda(1) \geq 5$, we deduce that $\mu(1) \lambda(1) \in \operatorname{cd}_{p}(G)$ cannot be a prime power whenever $\mu \in \operatorname{IBr}_{p}(G / K)$ with $\mu(1)>1$. Thus all irreducible $p$-Brauer characters of $G / K$ are linear. If $G / K$ is a $p^{\prime}$-group, then $\operatorname{Irr}(G / K)=$ $\operatorname{IBr}_{p}(G / K)$, so $G / K$ is abelian, which means that $K=G^{\prime}$. Otherwise, we see that $p=3, G / K$ has a Sylow $p$-subgroup $P / K$ of order $p$ and a normal Hall $p^{\prime}$-subgroup $C K / K$. By [23, Theorem 5.5] we have that $P / K \unlhd G / K$ and so $G / K=P / K \times C K / K$ is abelian as $C K / K \cong C / Z$ is abelian and $P / K$ is cyclic. Therefore, $K=G^{\prime}$ as required. 
Claim 4. If $G^{\prime}$ is nonabelian simple, then $C=\mathrm{Z}(G)$ is a $p^{\prime}$-group and Case (a) of the theorem holds.

We have $G^{\prime} \cap C=Z=1$ and hence $[G, C] \subseteq G^{\prime} \cap C=1$, which implies that $C \subseteq \mathrm{Z}(G)$. As $C=\mathrm{C}_{G}\left(G^{\prime}\right)$, we deduce that $\mathrm{Z}(G) \subseteq C$, so $C=\mathrm{Z}(G)$ in this case. Now by Claim $2, \mathrm{Z}(G)$ is a $p^{\prime}$-group. Recall that $G / \mathrm{Z}(G)$ is an almost simple group with simple socle $G^{\prime}$. If $G=G^{\prime} \mathrm{Z}(G)$, then since $G^{\prime} \cap \mathrm{Z}(G)=1$, we must have that $G=G^{\prime} \times \mathrm{Z}(G)$ and thus Case $(a)(i)$ holds. If $G / \mathrm{Z}(G)$ is not simple, then $G / \mathrm{Z}(G) \cong \mathrm{PSL}_{2}(8) \cdot 3$ with $p=3$ or $G / \mathrm{Z}(G) \cong \mathrm{PSL}_{2}\left(p^{f}\right)$ with $5 \leq p^{f} \leq 13$ by Corollary 3.8 . These give rise to Cases $(a)(i i)$ and $(a)(i i i)$, respectively.

Claim 5. If $G^{\prime}$ is not simple, then Case (b) holds.

Assume that $G^{\prime}$ is nonsimple. Then $G^{\prime} \cong \mathrm{SL}_{2}(5)$ and $p=5$. All statements of Case (b) follow from Claim 2 except for the last one. As $G / \mathrm{C}_{G}\left(G^{\prime}\right)$ is an almost simple group with simple socle $G^{\prime} / Z \cong \mathrm{PSL}_{2}(5)$, by Corollary 3.8 we have that either $G / C \cong \mathrm{PSL}_{2}(5)$ or $G / C \cong \mathrm{PGL}_{2}(5)$. If the latter case holds, then we are done. If the former case holds, then clearly $G / Z \cong C / Z \times G^{\prime} / Z$, $G^{\prime} \cap C=Z$ and $\left[G^{\prime}, C\right]=1$, so $G$ is a central product of $G^{\prime}$ and $C=\mathrm{C}_{G}\left(G^{\prime}\right)$.

The final claim. In this final claim, we will show that if the pair $(G, p)$ satisfies the conclusion of the theorem, then $\sigma_{p}(G)=1$.

Assume first that Case $(a)$ holds. Then $G^{\prime}$ is a nonabelian simple group with $\sigma_{p}\left(G^{\prime}\right)=1$ and $\mathrm{Z}(G)$ is a $p^{\prime}$-group.

Now if Case $(a)(i)$ holds, then $G=G^{\prime} \times \mathrm{Z}(G)$. In this case, we have that $\operatorname{cd}_{p}(G)=\operatorname{cd}_{p}\left(G^{\prime}\right)$ by [24, Theorem 8.21], so $\sigma_{p}(G)=\sigma_{p}\left(G^{\prime}\right)=1$. Assume that $G / \mathrm{Z}(G) \cong \mathrm{PSL}_{2}(8) \cdot 3, G^{\prime} \cong \mathrm{PSL}_{2}(8)$ and $\mathrm{Z}(G)$ is $p^{\prime}$-group, where $p=3$. Notice that $G / \mathrm{Z}(G)=G^{\prime}\langle\phi\rangle$, where $\phi$ is a field automorphism of $G^{\prime}$ of order 3. Using GAP, $\operatorname{cd}_{p}\left(G^{\prime}\right)=\{1,7,9\} ; G^{\prime}$ has exactly one irreducible $p$-Brauer character of degree 7 which is $\langle\phi\rangle$-invariant and three irreducible $p$-Brauer characters of degree 9 which are not invariant under $\phi$. Moreover, by [24, Theorem 8.21],

$$
\operatorname{IBr}_{p}\left(G^{\prime} \times \mathrm{Z}(G)\right)=\left\{\theta \times \lambda \mid \theta \in \operatorname{IBr}_{p}\left(G^{\prime}\right), \lambda \in \operatorname{IBr}_{p}(\mathrm{Z}(G))\right\} .
$$

Observe that all irreducible $p$-Brauer characters of $\mathrm{Z}(G)$ are $G$-invariant. Now if $\lambda \in \operatorname{IBr}_{p}(\mathrm{Z}(G))$ and $\theta \in \operatorname{IBr}_{p}\left(G^{\prime}\right)$ with $\theta(1)=1$ or 7 , then $\theta \times \lambda$ is $G$-invariant and thus by [24, Theorem 8.11], $\theta \times \lambda$ extends to $G$. If $\theta(1)=9$, then $\theta \times \lambda$ is not $G$-invariant and hence we must have that $(\theta \times \lambda)^{G} \in \operatorname{IBr}_{p}(G)$ as $\left|G: G^{\prime} \mathrm{Z}(G)\right|=$ 3. Therefore, we conclude that $\operatorname{cd}_{p}(G)=\left\{1,7,3^{3}\right\}=\operatorname{cd}_{p}\left(\mathrm{PSL}_{2}(8) \cdot 3\right)$. With similar argument, we can deduce that $\operatorname{cd}_{p}(G)=\operatorname{cd}_{p}\left(\mathrm{PGL}_{2}\left(p^{f}\right)\right)$ if $G / \mathrm{Z}(G) \cong$ 
$\mathrm{PGL}_{2}\left(p^{f}\right)$ with $p^{f}$ odd and $5 \leq p^{f} \leq 13$. Notice that in this case all irreducible $p$-Brauer characters of $\mathrm{PSL}_{2}\left(p^{f}\right)$ are $\mathrm{PGL}_{2}\left(p^{f}\right)$-invariant.

Finally, assume that Case (b) holds. Then $G^{\prime} \cong \mathrm{SL}_{2}(5), p=5 Z:=\mathrm{C}_{G}\left(G^{\prime}\right) \cap$ $G^{\prime}=\mathrm{Z}\left(G^{\prime}\right)$ and either $G$ is a central product of $G^{\prime}$ and $\mathrm{C}_{G}\left(G^{\prime}\right)$ or $G / \mathrm{C}_{G}\left(G^{\prime}\right) \cong$ $\mathrm{PGL}_{2}(5)$. Let $L:=G^{\prime} \mathrm{C}_{G}\left(G^{\prime}\right)$. In both cases, $L$ is a central product of $G^{\prime}$ and $\mathrm{C}_{G}\left(G^{\prime}\right)$. Writing $\operatorname{IBr}_{p}(Z)=\{1, \nu\}$. Since $G / Z$ has the structure as given in $(i)$ or $($ iii $)$ of Case $(a)$, we have that $\sigma_{p}(G / Z)=1$. Since $|G: L| \leq 2$, it suffices to show that all irreducible $p$-Brauer characters in $\operatorname{IBr}_{p}(L \mid \nu)$ have 2-power degrees. For any $\chi \in \operatorname{IBr}_{p}(L)$, there exist $\lambda \in \operatorname{IBr}_{p}\left(\mathrm{C}_{G}\left(G^{\prime}\right) \mid \nu\right)$ and $\varphi \in \operatorname{IBr}_{p}\left(G^{\prime} \mid \nu\right)$ such that $\chi=\lambda \cdot \varphi$. Using GAP, we can see that all irreducible $p$-Brauer characters in $\operatorname{IBr}_{p}\left(G^{\prime} \mid \nu\right)$ have 2-power degrees, so $\chi(1)=\lambda(1) \varphi(1)$ is a power of 2. In particular, all irreducible $p$-Brauer characters in $\operatorname{IBr}_{p}(L \mid \nu)$ have 2 -power degrees. The proof is now complete.

Proof of Theorem C. In view of $[9,12,13]$, we can assume that $G$ is nonsolvable. Let $K$ be the last term of the derived series of $G$ and let $L$ be a maximal normal subgroup of $K$. Then $K / L$ is a nonabelian simple group and all irreducible $p$-Brauer characters of $K / L$ have degrees 1 or primes. By Corollary 3.6, we have that $K / L \cong \mathrm{PSL}_{2}(p)$ with $p \in\{5,7\}$. In particular, $p$ is odd. Now the result follows from Theorem B. The proof is now complete.

\section{References}

[1] C. Bessenrodt and H. Weber, On p-blocks of symmetric and alternating groups with all irreducible Brauer characters of prime power degree, Journal of Algebra 320 (2008), no. $6,2405-2421$.

[2] C. Casolo and S. Dolfi, Prime divisors of irreducible character degrees and of conjugacy class sizes in finite groups, Journal of Group Theory 10 (2007), no. 5, 571-583.

[3] S. Dolfi, G. Navarro and P.H. Tiep, Primes dividing the degrees of the real characters, Mathematische Zeitschrift 259 (2008), no. 4, 755-774.

[4] A. Granville and K. Ono, Defect zero p-blocks for finite simple groups, Transactions of the American Mathematical Society 348 (1996), no. 1, 331-347.

[5] The GAP Group, GAP - Groups, Algorithms, and Programming, Version 4.5.6; 2012. http://www.gap-system.org.

[6] R. Guralnick, Subgroups of prime power index in a simple group, Journal of Algebra $\mathbf{8 1}$ (1983), no. 2, 304-311.

[7] G. Hiss and G. Malle, Low-dimensional representations of quasi-simple groups, LMS Journal of Computation and Mathematics 4 (2001), 22-63.

[8] D. Holt, On the local control of Schur multipliers, The Quarterly Journal of Mathematics. Oxford. Second Series 28 (1977), no. 112, 495-508. 
[9] B. Huppert, Solvable groups, all of whose irreducible representations in characteristic $p$ have prime degrees, Journal of Algebra 104 (1986), no. 1, 23-36.

[10] B. Huppert, Research in representation theory at Mainz (1984 - 1990), in Representation theory of finite groups and finite-dimensional algebras (Bielefeld, 1991), Progress in Mathematics, vol. 95, Birkhäuser, Basel, 1991, pp. 17-36.

[11] I.M. Isaacs, Character theory of finite groups, AMS Chelsea Publishing, AMS. Province, Rhode Island, 2006.

[12] I.M. Isaacs and D.S. Passman, A characterization of groups in terms of the degrees of their characters, Pacific Journal of Mathematics 15 (1965), 877-903.

[13] I.M. Isaacs and D.S. Passman, A characterization of groups in terms of the degrees of their characters. II, Pacific Journal of Mathematics 24 (1968), 467-510.

[14] C. Jansen, The minimal degrees of faithful representations of the sporadic simple groups and their covering groups, LMS Journal of Computation and Mathematics 8 (2005), $122-144$.

[15] U. Leisering, Solvable groups with p-modular character degrees of prime power, Archiv der Mathematik 55 (1990), no. 4, 324-332.

[16] M. Lewis and D. White, Connectedness of degree graphs of nonsolvable groups, Journal of Algebra 266 (2003), 51-76.

[17] F. Lübeck, Small degree representations of finite Chevalley groups in defining characteristic, LMS Journal of Computation and Mathematics 4 (2001), 135-169.

[18] G. Malle, Zeros of Brauer characters and the defect zero graph, Journal of Group Theory 13 (2010), no. 2, 171-187.

[19] G. Malle and A.E. Zalesskii, Prime power degree representations of quasi-simple groups, Archiv der Mathematik 77 (2001), no. 6, 461-468.

[20] O. Manz, Endliche auflösbare Gruppen, deren sämtliche Charaktergrade Primzahlpotenzen sind, Journal of Algebra 94 (1985), no. 1, 211-255.

[21] O. Manz, Endliche nicht-auflösbare Gruppen, deren sämtliche Charaktergrade Primzahlpotenzen sind, Journal of Algebra 96 (1985), no. 1, 114-119.

[22] O. Manz, R. Staszewski and W. Willems, On the number of components of a graph related to character degrees, Proceedings of the American Mathematical Society 103 (1988), 31-37.

[23] G.O. Michler, A finite simple group of Lie type has $p$-blocks with different defects, $p \neq 2$, Journal of Algebra 104 (1986), 220-230.

[24] G. Navarro, Characters and blocks of finite groups, LMS Lecture Note Series, vol. 250, Cambridge University Press, Cambridge, 1998.

[25] G. Navarro and P.H. Tiep, A reduction theorem for the Alperin weight conjecture, Inventiones Mathematicae 184 (2011), no. 3, 529-565.

[26] P.H. Tiep and W. Willems, Brauer characters of prime power degrees and conjugacy classes of prime power lengths, Algebra Colloquium 17 (2010), no. 4, 541-548.

[27] K. Zsigmondy, Zur Theorie der Potenzreste, Monatshefte für Mathematik und Physik 3 (1892), 265-284. 